\title{
Studies of Aggregated Nanoparticles Steering During Magnetic-Guided Drug Delivery in the Blood Vessels
}

\author{
Ali Kafash Hoshiara, , Tuan Anh Le ${ }^{\mathrm{a}}$, Faiz Ul Aminc, Myeong Ok Kimc, Jungwon Yoon ${ }^{\mathrm{a}, *}$ \\ ${ }^{a}$ School of Mechanical and Aerospace Engineering and ReCAPT, Gyeongsang National University, Jinju 660-701, Republic of Korea \\ ${ }^{b}$ Faculty of Industrial and Mechanical Engineering, Islamic Azad University, Qazvin Branch, Qazvin, Iran \\ ${ }^{c}$ Department of Biology and Applied of Life Science, Gyeongsang National University, Jinju 660-701, Republic of Korea
}

\begin{abstract}
Magnetic-guided targeted drug delivery (TDD) systems can enhance the treatment of diverse diseases. Despite the potential and promising results of nanoparticles, aggregation prevents precise particle guidance in the vasculature. In this study, we developed a simulation platform to investigate aggregation during steering of nanoparticles using a magnetic field function. The magnetic field function (MFF) comprises a positive and negative pulsed magnetic field generated by electromagnetic coils, which prevents adherence of particles to the vessel wall during magnetic guidance. A commonly used Y-shaped vessel was simulated and the performance of the MFF analyzed; the experimental data were in agreement with the simulation results. Moreover, the effects of various parameters on magnetic guidance were evaluated and the most influential identified. The simulation results presented herein will facilitate more precise guidance of nanoparticles in vivo.
\end{abstract}

Keywords: Targeted Drug Delivery (TDD), Nanorobotic, Aggregation, Magnetic Nanoparticles

\section{Introduction}

Micro-nano robotic systems for targeted drug delivery (TDD) based on non-invasive magnetic force actuation have been developed for guidance of particles in the vasculature. In this approach, nanoparticles are injected into a vein and circulate in the bloodstream. A magnetic force is used to steer the particles within the vasculature and guide them to the desired location. TDD requires generation of the optimal concentration of particles; i.e., a level greater than the minimum required and lower than the harmful dose, at the target location in vivo.

In traditional therapeutics, the whole body is exposed to high concentrations of toxic drugs, which, despite killing cancer cells, cause severe damage to healthy tissue. This can be overcome by using specially designed drugs to target only malignant tissues [1,2]. Although this method has certain benefits, it is non-optimal since only a tiny fraction of the drug administered reaches the target region. Therefore, to enhance treatment efficacy and maintain the optimal dose at the desired location, TDD systems have been developed [3].

TDD involves the following two steps: near-target injection and generation of a high concentration of drug at the site of interest [4]. However, in many cases, administration of a neartarget injection is medically hazardous. Consequently, guidance of micro-nano carriers within the vasculature requires development of a robotic platform.

*corresponding author at: School of Mechanical and Aerospace Engineering and ReCAPT, Gyeongsang National University, Jinju 660-701, Republic of Korea

Email address: jwyoon@gnu.ac.kr (Jungwon Yoon)
Magnetic resonance imaging (MRI)-based systems to steer microparticles in the vasculature have been described by others $[5,6]$. The requirement for more precise particle steering in the vasculature led to the development of magnetic-based microrobotic platforms $[7,8,9]$. Despite these benefits, however, the size factor prevents full interactions between particles and cells and prevents passage of the former through the blood-brain barrier (BBB) for TDD within the brain.

Therefore, we developed a novel nanorobotic platform with magnetic field function (MFF). The MFF is a simple varyingdirection magnetic gradient used to steer nanoparticles in the vasculature and overcome the particle-vessel adherence issue. The details of the system can be found in [10]. Nanoparticles up to $200 \mathrm{~nm}$ can cross the tight endothelial cells of the BBB $[11,12]$. Furthermore, the external magnetic field can improve transport of magnetic nano particles (MNPs) through cell barrier and $\mathrm{BBB}$ by the endocytosis process $[13,14]$. Besides, the evidence of clustered MNPs has been found in the mouse brain after BBB crossing [14]. Similarly, we also reported that magnetic particles of $770 \mathrm{~nm}$ (mean diameter) can cross the normal BBB when subjected to external electromagnetic fields [15]. Despite the encouraging results, in the real world the aggregation phenomenon (which was not considered in the model) reduces system performance and should be considered during the design stage of magnetic guidance systems.

The aggregation of microparticles in Y-shaped vessels has been investigated experimentally [16], and an aggregation simulation platform has been developed to increase the efficacy of the magnetic force. To utilize aggregation, it has been suggested that a magnetic force should be applied parallel to the

October 20, 2016 
direction of blood flow, which results in movement of aggregated particles with blood flow or due to application of a constant magnetic field $[17,18]$. Although these studies increased our understanding of the aggregation phenomenon, aggregations orthogonal to the direction of flow may result in clogging of vessel branches, which is clearly not desirable for drug delivery to the brain. Moreover, in practice, particle-vessel adherence, which occurs due to low blood-flow velocity near the vessel walls and could explain the low targeting efficiency upon application of a constant magnetic gradient is not considered.

Therefore, to both consider the effects of aggregation and overcome the adherence problem using an MFF, we have developed a comprehensive platform for simulation of particle steering. Experimental studies were carried out to verify the effectiveness of the platform.

In this paper, first, the aggregation in real drug-delivery environments has been demonstrated and the results of in vivo experiments are presented. Then, a simulation model of a Yshaped channel was developed to investigate the steering performance of aggregated particles under the MFF in the channel; experimental studies were conducted to verify the results. Finally, the parameters with the greatest influence on aggregation were determined to evaluate guidance using the MFF.

\section{The magnetic based guidance architecture}

To steer magnetic nano particles (MNPs) in the vesicular a magnetic guidance system which is illustrated in Fig. (1) has been used. The magnetic force adjusts the particle position and guides it through the correct outlet.

Electromagnetic actuators generate a force adequate for nanoparticle steering within the vasculature. The electromagnetic actuator comprises two sets of coils (7,000 turns and diameter $\mathrm{d}=1.0 \mathrm{~mm}$ ) with a core to increase the magnetic field density (cobaltiron alloy Vacoflux 50). Currents of up to $17 \mathrm{~A}$ (gradient filed strength $7.9 \mathrm{~T} / \mathrm{m}$ ) can be applied to the system.

The optimum conditions for MFF vary according to blood vessel size, blood flow velocity, particle size, and the magnetic field applied. This time varying on-off function changes the direction of the field function and guides particles to the desired outlet. The details of MFF are provided elsewhere [10, 15].

\subsection{Effects of the aggregation on the targeting performance}

The introduced system was used to guide nanoparticles with $350 \mathrm{~nm}$ mean diameters to the mouse brain. These fluorescent carboxyl magnetic nanocontainers can be used to track the MNPs in the brain.

The particles were injected into the tail of the mice, which were then exposed to different magnetic field functions to experimentally find the optimum condition. To study the drug uptake, experiments in absence of the magnetic field, with a constant magnetic field and with a magnetic field function (MFF) have been performed. In absence of the magnetic force no evidence of nanoparticles has been found in the brain. To study constant magnetic field, the input current increased from $1 \mathrm{~A}$ to $3 \mathrm{~A}$ which resulted in higher magnetic force and lead to more

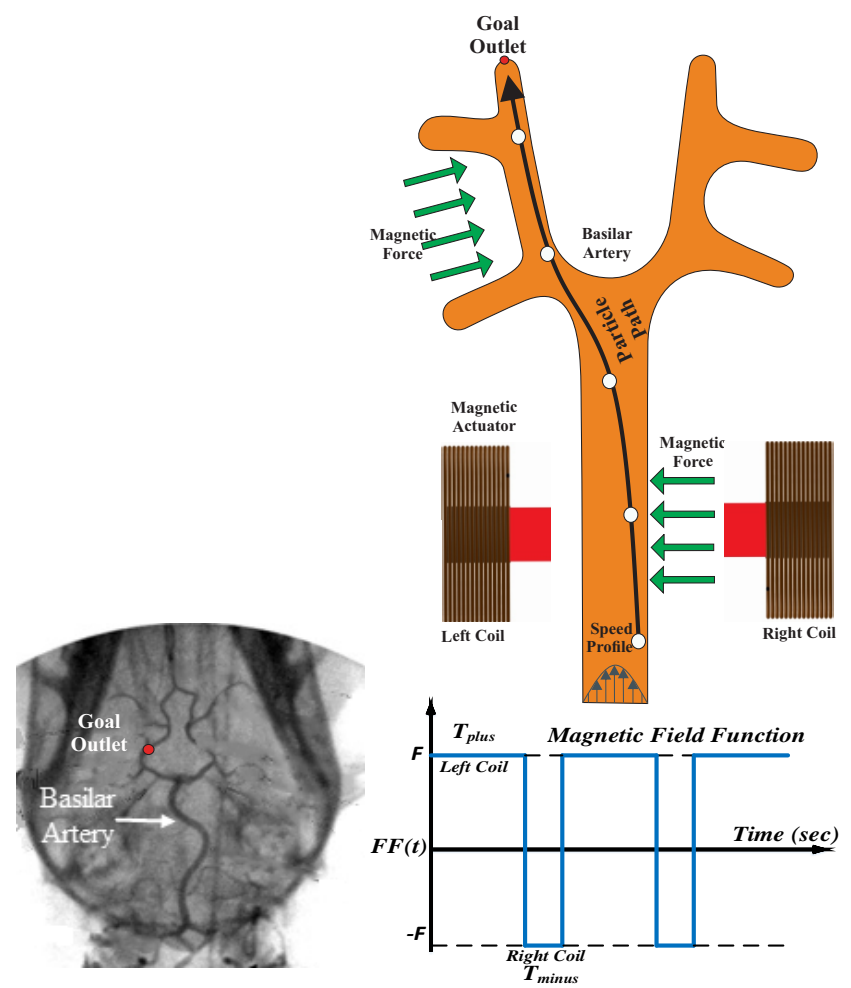

Figure 1: The mechanism of nanoparticle steering within blood vessels with the magnetic field function $(F F(t))$ guidance.

uptake into the brain. With the MFF the rate of particle crossing BBB has been improved significantly. It has been observed that the MFF could solve the vessel-particle adherence issue and successfully guided the particles to the cortical areas of the brain [15].

To study the MFF, the current of the coils has been changed between 1 to $3 \mathrm{~A}$ and the frequency of the magnetic field function sets as $0.25,0.5$ and $1 \mathrm{~Hz}$. Despite the improvement in the $\mathrm{BBB}$ crossing, we have observed that the particles aggregated in the brain after crossing BBB as shown in Fig. $2 \mathrm{~A}$ and B.

As the aggregation phenomenon was not considered during the initial design of MFF, it reduced the performance of the system. The particle distribution analysis indicated that, by using the optimum conditions in [15], the particle density on the left and right sides of the brain was almost equal; i.e., the MFF was ineffective for targeting, even though it helped the magnetic particles pass through the BBB.

The microscopic image and particle distribution demonstrates existence of particle aggregation in Fig. 2. Considering the detrimental effects of aggregation both in BBB crossing and targeting, the simulation platform should be developed to study the effects of aggregation. However, the mechanism of BBB crossing for the aggregated nanoparticles is not in the scope of this paper and will be studied separately with consideration of molecular dynamic (MD) based simulations [19]. The simulation platform in this paper has been developed to improve the steering performance of aggregated MNPs. 

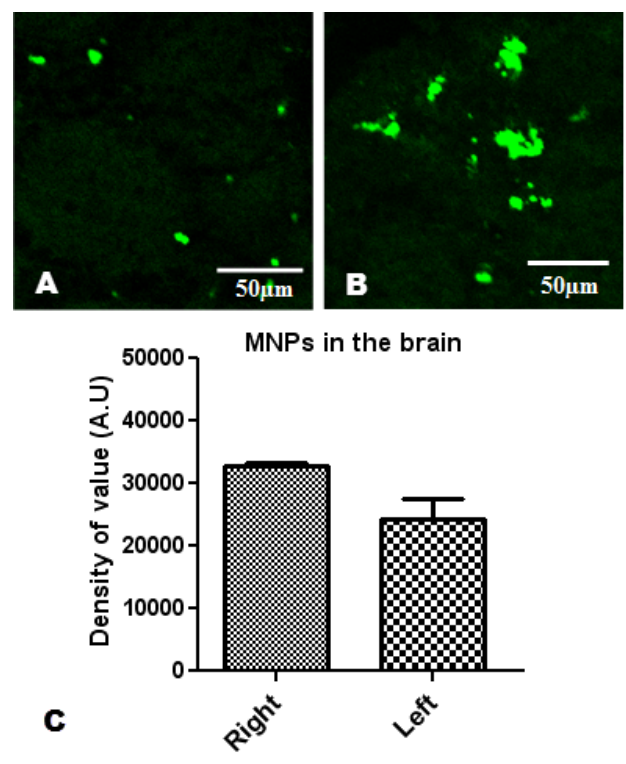

Figure 2: Evidence of aggregation of MNPs after crossing BBB in the left (A) and right (B) brain hemispheres of a test subject (confocal laser scanning microscope image), and the particle distribution therein.

\section{Dynamic Forces Governing TDD}

The major forces governing the aggregation phenomenon were investigated, an overview of which is provided in this section. Many parameters presented in this section will be used throughout the manuscript and any change in these parameters will be discussed in subsequent sections. Forces depicted in Fig. 3 have been taken into account, and the Newtonian dynamic model is presented as:

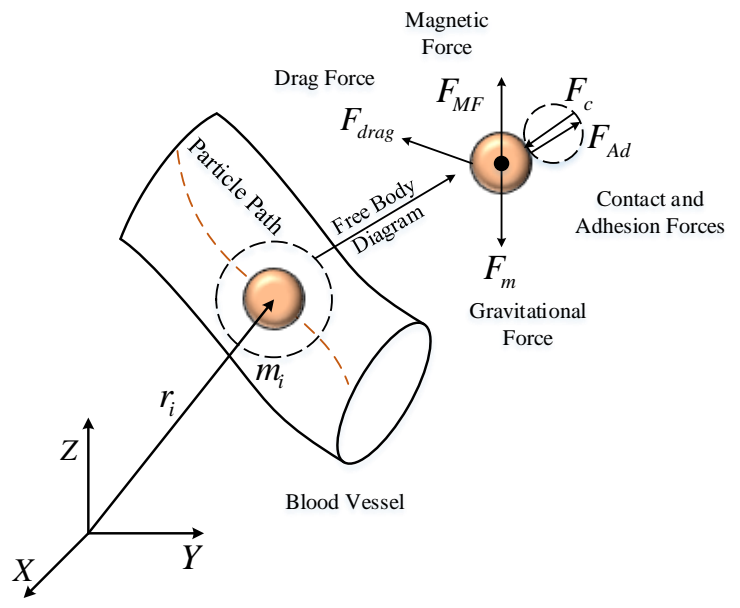

Figure 3: Free-body diagram of the dominant forces.

$$
m_{i} \frac{d v_{i}}{d t}=F_{M F}+F_{\text {drag }}+F_{c}+F_{A d}+F_{m}
$$

where the index $i$ indicates particle $i . v_{i}$ is the particle velocity, $F_{M F}$ is the magnetic force, $F_{\text {drag }}$ is the hydrodynamic drag force, $F_{m}$ is the gravitational force, $F_{c}$ is the contact force and $F_{A d}$ is the adhesion force. To use Newtonian mechanics, particles are considered to be large enough to exclude the Brownian effect [18].

The magnetic force is the only actuation source in the nanorobotic platform. Basically, due to permeability differences, the nanoparticles are subjected to a force in a magnetic field [10], which has been modeled as:

$$
F_{M F}=F F(t)\left[\frac{4}{3} \pi \mu_{1} R^{3} M_{\mathrm{sat}} \nabla H\right]
$$

where $M_{\text {sat }}$ is the magnetic polarization, $\nabla H$ is the gradient of magnetic intensity, $\mu_{1}$ is the permeability of the free space and the particles are considered uniform spheres of radius $R$. $F F(t)$ is the time varying function which has been illustrated in the Fig 1 and used to change the direction of the magnetic field.

Magnetic nanoparticles are super magnets that exhibit hysteresis-free behavior, which enables them to act as perfect magnets. During aggregation, the dipole force has two main effects a negligible contribution to the magnetic intensity $(H)$ and a major influence on particle-particle adherence after collision. To model the magnetic moment, a system of coupled equations must be solved. First, the magnetic intensity for $i^{t h}$ particle with respect to $j^{\text {th }}$ is as follows:

$$
H_{i j}=\frac{\mu_{0}}{4 \pi}\left[\frac{\left.3\left(m_{j} \cdot r_{i j}\right) r_{i j}\right)}{r_{i j}^{5}}-\frac{m_{j}}{r_{i j}^{3}}\right]
$$

where subscripts $i$ and $j$ denote the particles, $r$ is the distance between the particles and $m$ is the magnetic moment. The total magnetic intensity for the particle of interest is given as:

$$
H=H_{\text {ext }}+\sum_{j}^{N} H_{\text {dip }}
$$

where $H_{\text {ext }}$ is the external magnetic intensity, and $\sum$ adds the accumulated effect of other particles. Finally, the total magnetic moment can be described as:

$$
M_{i}=\frac{4 \pi}{\mu_{0}} \frac{\mu_{p}-1}{\mu_{p}+2} \frac{d_{i}^{3}}{8} H_{i}
$$

where $\mu_{p}$ is the permeability of the particle and $d$ is its diameter. Solving this system of $3 \mathrm{~N}$ coupled equations yields the magnetic moment $[17,18]$.

The drag (hydrodynamic) force of a sphere based on the Stokes law is:

$$
F_{\text {drag }}=-6 \pi \eta R\left(v_{p}-v_{f}\right)
$$

where $v_{p}$ and $v_{f}$ are the particle and fluid velocities, respectively, $R$ is the particle radius and $\eta$ is the fluid viscosity.

The gravity and bouncy forces determine the gravitational force, as follows:

$$
F_{m}=\frac{4}{3} \pi R^{3}\left(\rho_{p}-\rho_{b}\right) G
$$

where $R$ is the particle radius, and $\rho_{p}$ and $\rho_{b}$ are the particle and blood densities, respectively. 
Contact forces are generated as a result of particle-particle or particle-vessel collisions. The Hertzian contact model can be expressed as:

$$
F_{c}=k \delta^{\frac{3}{2}} \stackrel{\text { If }}{\leftrightarrow} d<R_{i}+R_{j}
$$

where $d$ is the particle-particle distance, $R_{i}$ is the $i^{\text {th }}$ particle radius, $R_{j}$ is the $j^{\text {th }}$ particle radius, $k$ is the spring constant, and $\delta$ is deformation.

Although nanoparticle coating can decrease the effects of adhesion, this force has been introduced since the sticking issue could not be entirely solved by coating and the parameters of adhesion model can be adjust based on the coating characteristics [20]. The adhesion force is a function of the adhesive energy and deformation area. $F_{A d}$ is modeled as:

$$
F_{A d}=\tau \pi\left(\frac{3 F_{c} R}{4 E^{*}}\right)^{\frac{2}{3}}
$$

where $\tau$ is the adhesive energy (a constant), $R$ is the equal radius ( $\left.R=\frac{R_{1} R_{2}}{R_{1}+R_{2}}\right), F_{c}$ is the contact force, and $E^{*}$ is the equal elasticity module. The equal elasticity module $\left(E^{*}\right)$ is presented as:

$$
\frac{1}{E^{*}}=\frac{1-v_{1}^{2}}{E_{1}}+\frac{1-v_{2}^{2}}{E_{2}}
$$

where the subscripts 1 and 2 denote particle numbers, $E$ is the elasticity module and $v$ is Poissons ratio. The opposite nature of the contact force (separation) and adhesion force (connection) results in a competition with two possible outcomes; particle aggregation or particle detachment.

\section{Results and Discussion}

In this section, a two-dimensional model based on discrete element method (DEM) simulation for guidance of aggregated nanoparticles in a Y-shaped blood vessel is described. This section is divided into three subsections. In the first, a computational model capable of generating meaningful physical results is described. The second subsection compares the simulation results with those obtained experimentally to validate the accuracy of the model. The final subsection presents particlesteering performance according to various parameters.

\subsection{Aggregation model}

Fig. 4 shows a flowchart of the aggregation model. First, the velocity profile data were extracted using the COMSOL simulation package. Then, the forces were computed based on the modeling information provided in the previous section. The contact condition in each time step determined particle integration, and the magnetic and drag forces governed particle movements. The governing dynamic equation (Eq. 1) at each time step describes particle position. Following aggregation, the particles moved at different velocities, so the aggregated particle velocity was calculated. Based on the field function properties $\left(T_{\text {plus }}, T_{\text {minus }}\right)$ the direction of the magnetic field changed, causing particle movement within the vessel. Finally, the number of particles reaching the correct outlet was determined.

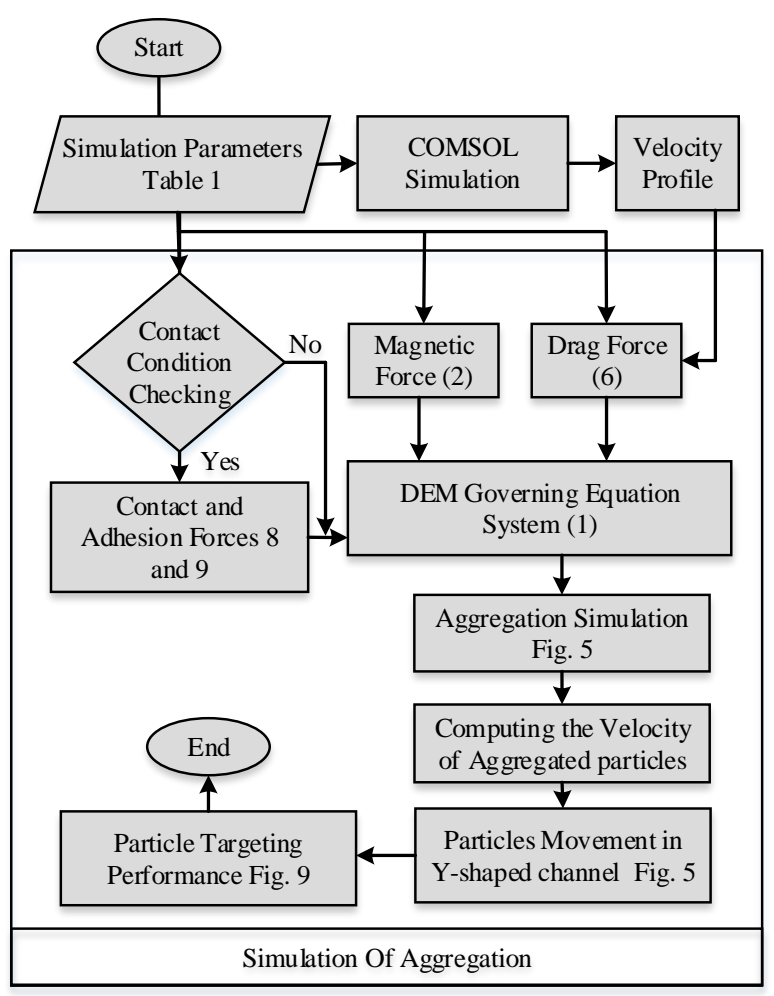

Figure 4: Flowchart of simulation of aggregation in a Y-shaped blood vessel.

\subsubsection{Velocity profile within blood vessels}

Since blood vessel bifurcations resemble Y-shaped channels, a Y-shaped channel was used as the model in the simulations. The Y-shaped channel consists of one inlet and two outlets of constant diameter $d_{w}$. A steady creeping flow enters through the inlet and exits via the outlets. The flow is considered to be steady state and the CFD module of the COMSOL package was used to extract the blood velocity profile data based on the geometry of the vessel.

\subsubsection{Simulation of the aggregation}

The aggregation phenomenon during MNP magnetic guidance in a Y-shaped channel were simulated, taking into consideration the governing dynamics and using the nominal values provided in Table (1). The magnetic force (the actuation force) was simulated based on the model introduced in the previous section and was considered a body force. In the case of a particle-particle or particle-vessel contact, the contact and adhesion forces were applied using Eqs. (8) and (9). The drag force was determined using the blood velocity profile information. All of the forces were incorporated into the governing equation (1) and a system of ODEs was formed.

In this simulation, 225 particles of $400 \mathrm{~nm}$ radius were used. Thus, in each time step, 900 ODE equations must be solved. Resolution of this ODE system using the RungeKutta method resulted in identification of the aggregation mechanism (Fig. 5).

The particles aggregated near the inner wall of the vessel in a parabolic curve shape due to the combined effect of the initial position of the particles and the blood velocity profile. More- 
Table 1: Parameters used for simulation of TDD.

\begin{tabular}{c||ccc}
\hline Category & Parameter & Value & Unit \\
\hline \multirow{2}{*}{$\begin{array}{c}\text { Particle } \\
\text { Properties }\end{array}$} & $d$ & 800 & $(\mathrm{nM})$ \\
\cline { 2 - 4 } & $\rho_{p}$ & 6450 & $\left(\mathrm{Kg} / \mathrm{M}^{3}\right)$ \\
\hline \multirow{2}{*}{ Process } & $M_{\text {sat }}$ & 2 & $(\mathrm{~T})$ \\
\hline \multirow{3}{*}{$\begin{array}{c}\text { Environmental } \\
\text { Effects }\end{array}$} & $\nabla H$ & 0.30 & $\left(\mathrm{MA} / \mathrm{m}^{2}\right)$ \\
\cline { 2 - 4 } & $\rho_{b}$ & 1050 & $\left(\mathrm{Kg} / \mathrm{M}^{3}\right)$ \\
\hline
\end{tabular}

over, particles aggregated in a column shape. There are two possible scenarios for the particles: if the contact force surpasses the total effects of the dipole and adhesion forces, particles change direction without aggregating; otherwise, they form a column-shaped assembly.

\subsubsection{Guidance of aggregated particles using the MFF}

The MFF overcomes the adherence problem and facilitates particle movement within blood vessels by changing the direction of the magnetic force. The magnetic and drag forces govern the dynamics of the movement of particles inside the vessel. The magnitude of the magnetic and drag forces varies with the number of aggregated particles in a column. Therefore, particle velocity varies according to aggregation size. To simulate this effect, the velocity of each column was computed taking into consideration the size and number of aggregated particles.

The particles move inside the vessel under the guidance of the applied MFF and reach the correct outlet. Fig 5 shows the aggregation simulation within Y-shaped channel. Initially, single particles integrate and generate aggregates $\left(t_{0}\right)$. Then, the column-shaped aggregates move based on the designed MFF at different velocities and reach the bifurcation. Finally, as shown in Fig 5, particles reach the correct outlet at $T_{\text {Final }}$. Using this simulation model, the performance of the MFF can be demonstrated by computing the number of particles that reach the correct outlet.

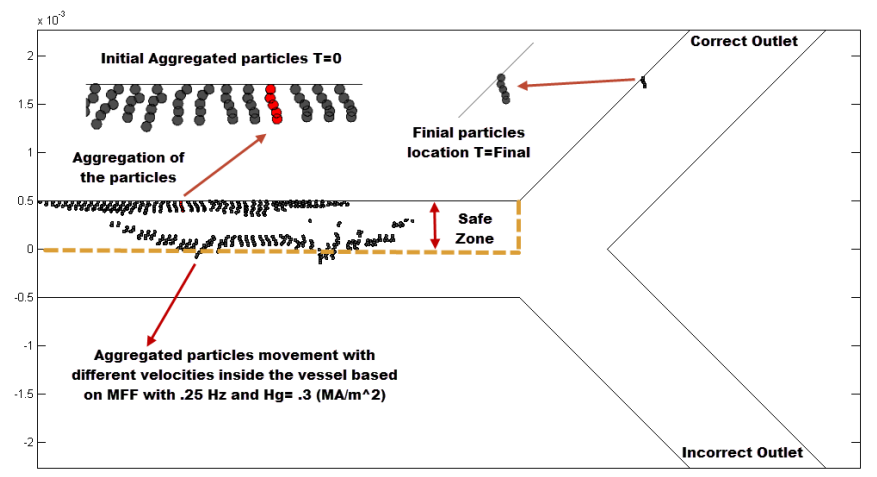

Figure 5: Steering of the aggregated particles in a Y-shaped channel with the MFF.

\subsection{Experimental verification of the computational platform}

At the macroscopic level, the magnetic silica particles were exposed to a magnetic field, and the results demonstrated an aggregation pattern identical to that suggested by the simulations. At the microscopic level, the particles aggregated in a column-shaped pattern [18].

To evaluate the accuracy of the simulation model, magnetic silica particles (800 $\mathrm{nm}$ diameter) were guided within a Yshaped channel using magnetic actuators. The experimental setup is shown in Fig. (6).

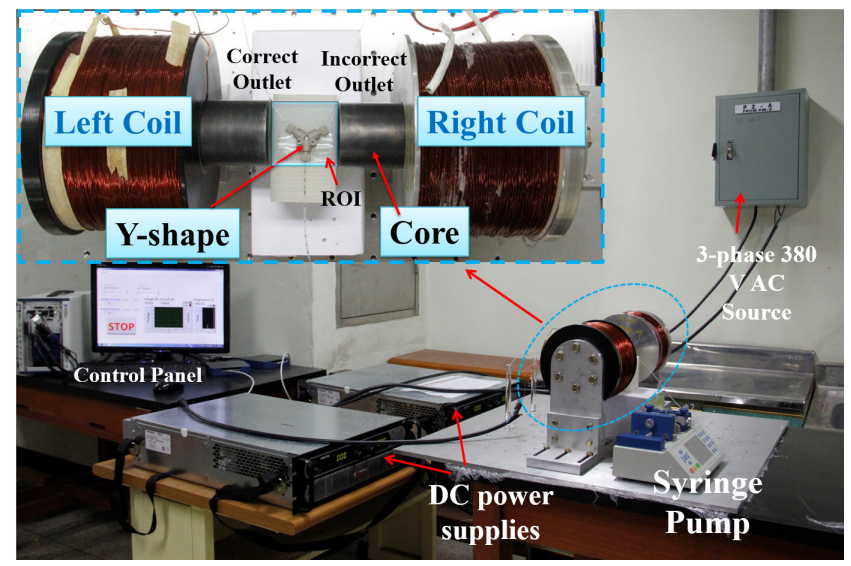

Figure 6: The Y-shaped channel used in this study.

To precisely steer particles in the channel, we assumed that the particles that remain in the safe zone (the area between the upper bound of the vessel and the midpoint of the vessel) reach the correct outlet (Fig. 5). Therefore, the magnetic force should be designed in a way that forces particles to remain in the safe zone. A camera was used to monitor the positions of particles in the channel. These position data have been used to identify the time needed for particles to move from vessel boundary to the safe zone limit.

The magnetic force is a function of the gradient of the magnetic intensity $\left(H_{g}\right)$, so we altered $H_{g}$ and calculated the maximum time required for particles to reach the safe zone limit; the results are presented in Fig.(7).

In the computational platform, the time for aggregated particles to reach the same position in the vessel depends on the number of aggregated particles. Therefore, simulations using different numbers of aggregated particles were performed, and the results are shown in Fig. (7). The simulation results are in acceptable agreement with those obtained experimentally. Therefore, the proposed model predicts particle movement precisely.

\subsection{Factors that influence nanoparticle guidance in blood ves- sels}

Since the goal of TDD is to send the maximum number of particles to the correct outlet, the number of particles reaching the correct outlet defines the system performance. The efficacy of the process depends on environmental, geometric and process parameters. The parameters with the greatest effects are 


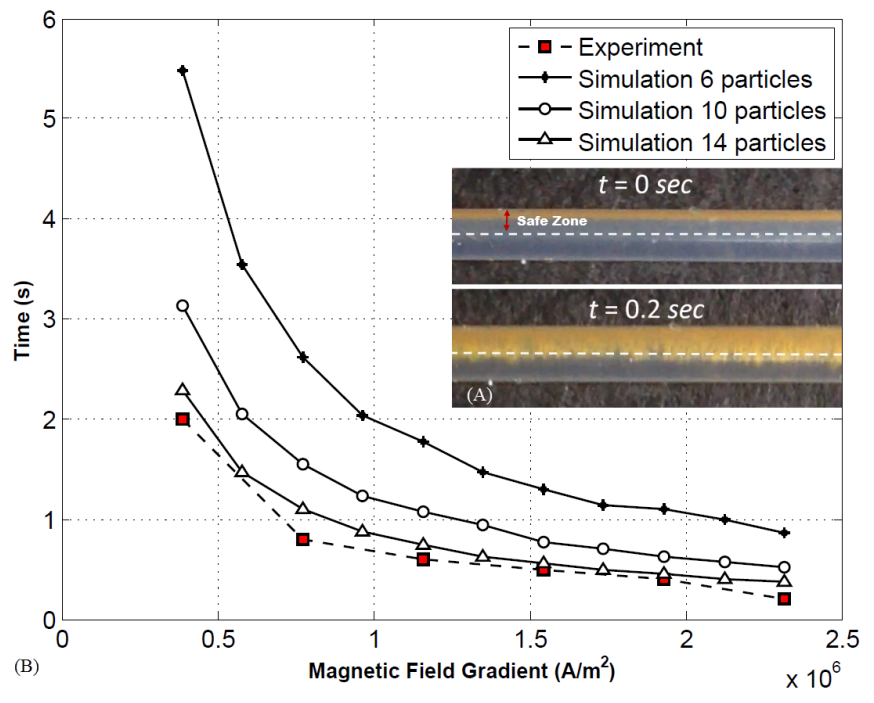

Figure 7: A) Camera data on aggregated particle movement in the safe zone (MFF $H_{g}=2.3 \mathrm{MA} / \mathrm{m}^{2}, 0.25 \mathrm{~Hz}$ ) B) Particle movement in the vessel based on different magnetic field gradients $\left(H_{g}\right)$ as determined by simulation and experimentally (the information from Table 1 is used for both experiment and simulation).

particle size, magnetic intensity gradient and blood-flow velocity; these were varied within the ranges shown in Table (2). To evaluate system performance, the relationships between the various parameters and system performance were determined by conducting several simulations.

The effects of blood velocity on the percentage of particles reaching the correct outlet are shown in Fig. (8). The time required for maximum performance to be achieved decreases with increasing blood-flow velocity.

Table 2: Parameters found to affect TDD performance.

\begin{tabular}{c||cc} 
& Range of the parameter change & \\
\hline$H_{g}$ & 0.30 & $\frac{M A}{m^{2}}$ \\
$v_{b}$ & $10,20,30,40,50,60,70,80,90,100$ & $\frac{m m}{s}$ \\
$d_{p}$ & $700,800,900,1000$ & $\mathrm{~nm}$
\end{tabular}

The magnetic force (the actuation force) is a function of two parameters: the magnetic intensity gradient and the particle size. The aggregation influences the magnitude of the magnetic force and affects the governing dynamics. Therefore, to present its effects the force factor [10] is defined as a new parameter which composed of $H_{g}$ (magnetic field gradient) and $d_{p}$ (particle diameter) as $R_{f}=H_{g} . d_{p}^{3}$. In the simulation magnitude of the magnetic field gradient is considered as a constant $H_{g}=.307 \mathrm{MA} / \mathrm{m}^{2}$ and $d_{p}$ varies according to Table 2 . The geometry of the vessel is considered as vessel diameter $d_{v}=1 \mathrm{~mm}$ and vessel length $L_{v}=10 \mathrm{~mm}$ and four blood velocity condition $V_{b}=[100,25,10,2.5] \mathrm{mm} / \mathrm{s}$ has been considered. For each condition, we have performed a simulation and the results have been illustrated in the Fig. 9.

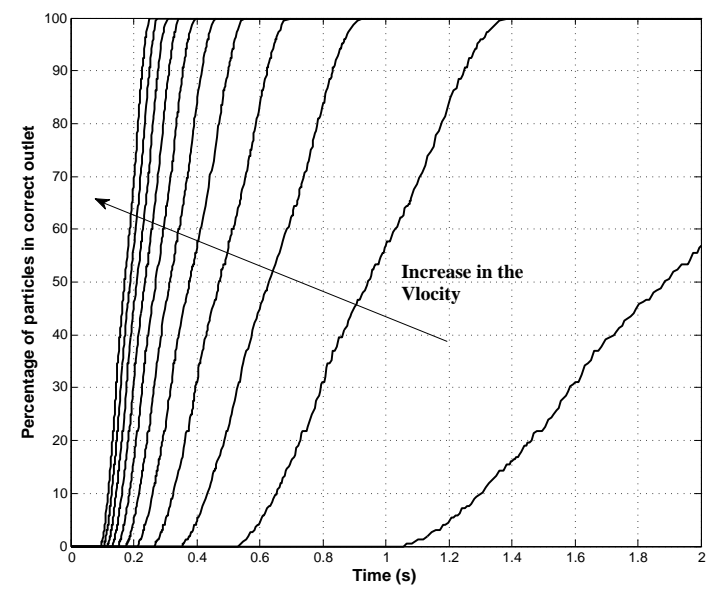

Figure 8: Results of simulation of steering performance according to particle velocity with a MFF duty cycle of $0.25 \mathrm{~Hz}$ with equal $T_{\text {plus }}-T_{\text {minus }}$ (see Fig. 1) and $H_{g}=0.3 \mathrm{MA} / \mathrm{m}^{2}$.

The Fig. 9 presents the particle distribution pattern. In absence of the magnetic force, number of particles reaching to the correct outlet is 50 percent as initially the particles are equally distributed in the tube. As expected, the magnetic force increased with aggregation so the number of particles reaching the correct outlet is higher than the previous estimation [10]. In the previous analysis particles considered to be single and the aggregation effects have not been included so the magnetic force in the high blood velocity was ineffective as it did not have enough power to influence. However, the aggregated particles have a better response with respect to magnetic force.

The Fig.9 C and D presents the particle distribution in the low flow velocities. In the low velocity the sticking issue of nanoparticles occurs. The field function concept has been introduced to solve this problem. We have found that the sticking issue has been solved and the evidence of particles have been found in the mouse brain (details in [15]). The simulation results presented in the Fig. $9 \mathrm{C}$ and $\mathrm{D}$ confirm that particle sticking issue has been solved. Nevertheless, the growth in the number of particles reaching the incorrect outlet is evident. The main reason for this trend is the increase of magnetic force with aggregation. The force factor clearly demonstrates this trend as the growth in the force factor leads to a rise in the number of particles reaching the incorrect outlet which is the evidence of the system uncertainty. This analysis is in agreement with our recent particle distribution analysis experiments (Fig.2).

The aggregation phenomenon also could explain the reason of the remaining particles increase in the Fig.9 D. The aggregation leads to a growth in the magnetic force which at the same time leads to an increase in the vertical velocity of particles. The rapid vertical movement of particles prevent the particle movement with the flow (horizontal movement) and results in a rise in the remaining particles. Consequently, although the sticking issue has been solved, the particles remain in the vessel as the magnetic force was not adjusted adequately. 


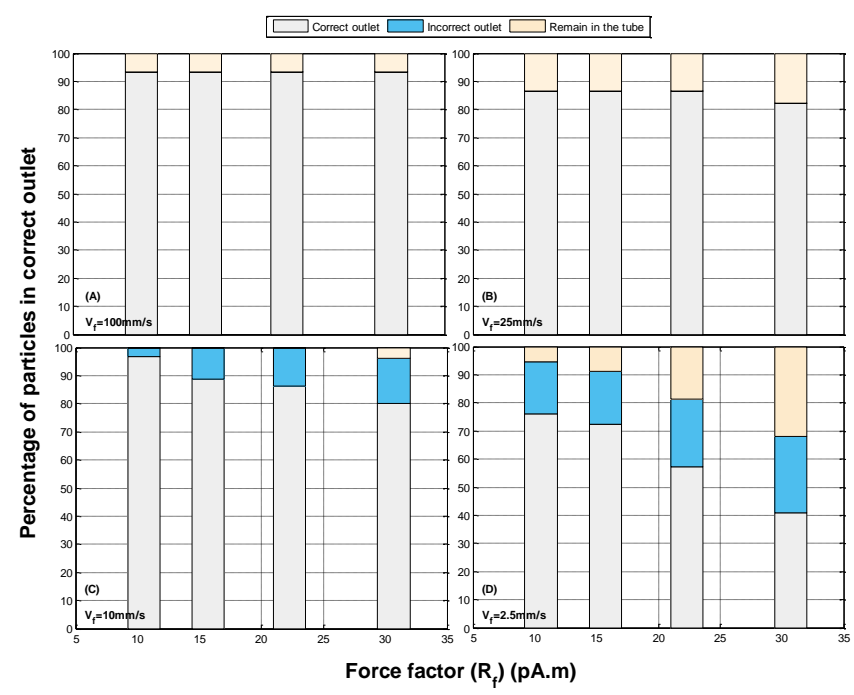

Figure 9: Results of steering performance simulation for a Y-shaped vessel with $d_{v}=1 \mathrm{~mm}, L_{v}=10 \mathrm{~mm}$ and MFF duty cycle of $0.25 \mathrm{~Hz}$ with equal $T_{\text {plus }}$ $T_{\text {minus }}$ and $R_{f}=[10.5,15.7,22.3,30.7] p A . m$ and for four velocity conditions A) $V_{b}=100 \mathrm{~mm} / \mathrm{s}$, B) $V_{b}=25 \mathrm{~mm} / \mathrm{s}$, C) $V_{b}=10 \mathrm{~mm} / \mathrm{s}$ and D) $V_{b}=2.5 \mathrm{~mm} / \mathrm{s}$

\section{Conclusion}

Although aggregation effectively improves the actuation force, the phenomenon influences TDD performance markedly. Therefore, investigation of aggregation can facilitate the improvement of targeting efficiency.

The proposed aggregation model simulates steering performance in a Y-shaped vessel by means of an MFF, and the results were in agreement with those obtained experimentally. Moreover, velocity, magnetic intensity and particle size were found to influence steering efficiency. The results will facilitate design of a MFF to enable precise guidance of nanoparticles within the vasculature.

So long as they are retained within the safe zone by the MFF, particles move rapidly towards the correct outlet. However, the MFF must be modified to enable precise guidance with respect to different force factor $R_{f}$ (the particle size and gradient of magnetic intensity). Moreover, the results of aggregated particles steering justify the particle distributions in mouse experiments.

Future work will expand the simulations for the multiple branching and more realistic vessels. Besides, the design of the field function will be improved to prevent or reduce the aggregation effects.

\section{Acknowledgment}

This research was supported by the Pioneer Research Center Program through the National Research Foundation of Korea funded by the Ministry of Science, ICT and Future Planning (2012-0009524) and in part by NRF2012R1A2A2A01047344 and 2014R1A2A1A11053989.

\section{References}

[1] N. Bertrand, J. Wu, X. Xu, N. Kamaly, O. C. Farokhzad, Cancer nanotechnology: The impact of passive and active targeting in the era of modern cancer biology, Advanced Drug Delivery Reviews 66 (2014) 2 25.

[2] S. D. Steichen, M. Caldorera-Moore, N. A. Peppas, A review of current nanoparticle and targeting moieties for the delivery of cancer therapeutics, Eur J Pharm Sci 48 (3) (2013) 416-427.

[3] F. Dilnawaz, S. K. Sahoo, Therapeutic approaches of magnetic nanoparticles for the central nervous system, Drug Discovery Today 20 (10) (2015) 1256-1264.

[4] S. Merino, C. Martín, K. Kostarelos, M. Prato, E. Vázquez, Nanocomposite hydrogels: 3d polymer-nanoparticle synergies for on-demand drug delivery, ACS nano 9 (5) (2015) 4686-4697.

[5] J.-B. Mathieu, S. Martel, Magnetic microparticle steering within the constraints of an mri system: proof of concept of a novel targeting approach, Biomedical microdevices 9 (6) (2007) 801-808.

[6] S. Martel, J.-B. Mathieu, O. Felfoul, A. Chanu, E. Aboussouan, S. Tamaz, P. Pouponneau, L. Yahia, G. Beaudoin, G. Soulez, et al., A computerassisted protocol for endovascular target interventions using a clinical mri system for controlling untethered microdevices and future nanorobots, Computer Aided Surgery 13 (6) (2008) 340-352.

[7] H. Choi, K. Cha, J. Choi, S. Jeong, S. Jeon, G. Jang, J. oh Park, S. Park, \{EMA\} system with gradient and uniform saddle coils for $3 \mathrm{~d}$ locomotion of microrobot, Sensors and Actuators A: Physical 163 (1) (2010) 410 417.

[8] S. Jeong, H. Choi, J. Choi, C. Yu, J. oh Park, S. Park, Novel electromagnetic actuation (ema) method for 3-dimensional locomotion of intravascular microrobot, Sensors and Actuators A: Physical 157 (1) (2010) 118 -125 .

[9] M. P. Kummer, J. J. Abbott, B. E. Kratochvil, R. Borer, A. Sengul, B. J. Nelson, Octomag: An electromagnetic system for 5-dof wireless micromanipulation, IEEE Transactions on Robotics 26 (6) (2010) 1006-1017.

[10] M. D. Tehrani, J.-H. Yoon, V. Kim, J. Yoon, A novel scheme for nanoparticle steering in blood vessels using a functionalized magnetic field, Biomedical Engineering, IEEE Transactions on 62 (1) (2015) 303-313.

[11] C. Pardesh, P. Rajput, V. Belgamwar, A. Tekade, P. Ganesh, C. Kapil, S. Abhjeet, Solid lipid based nanocarriers: an overview, Acta Pharmaceutica 62 (4) (2012) 433-472.

[12] J. V. Georgieva, D. Kalicharan, P.-O. Couraud, I. A. Romero, B. Weksler, D. Hoekstra, I. S. Zuhorn, Surface characteristics of nanoparticles determine their intracellular fate in and processing by human blood-brain barrier endothelial cells in vitro, Molecular Therapy 19 (2) (2011) 318325.

[13] K. A. Min, M. C. Shin, F. Yu, M. Yang, A. E. David, V. C. Yang, G. R. Rosania, Pulsed magnetic field improves the transport of iron oxide nanoparticles through cell barriers, ACS Nano 7 (3) (2013) 2161-2171.

[14] S. D. Kong, J. Lee, S. Ramachandran, B. P. Eliceiri, V. I. Shubayev, R. Lal, S. Jin, Magnetic targeting of nanoparticles across the intact bloodbrain barrier, Journal of Controlled Release 164 (1) (2012) 49 - 57.

[15] T. D. Do, F. U. Amin, Y. Noh, M. O. Kim, J. Yoon, Guidance of magnetic nanocontainers for treating alzheimer's disease using an electromagnetic, targeted drug-delivery actuator, Journal of Biomedical Nanotechnology 12 (3) (2016) 569-574.

[16] J.-B. Mathieu, S. Martel, Steering of aggregating magnetic microparticles using propulsion gradients coils in an mri scanner, Magnetic Resonance in Medicine 63 (5) (2010) 1336-1345.

[17] P. Vartholomeos, C. Mavroidis, Simulation platform for self-assembly structures in mri-guided nanorobotic drug delivery systems, in: Robotics and Automation (ICRA), 2010 IEEE International Conference on, IEEE, 2010, pp. 5594-5600.

[18] P. Vartholomeos, C. Mavroidis, In silico studies of magnetic microparticle aggregations in fluid environments for mri-guided drug delivery, Biomedical Engineering, IEEE Transactions on 59 (11) (2012) 3028-3038.

[19] A. Shamloo, M. Z. Pedram, H. Heidari, A. Alasty, Computing the blood brain barrier (bbb) diffusion coefficient: A molecular dynamics approach, Journal of Magnetism and Magnetic Materials 410 (2016) 187 - 197.

[20] B. Issa, I. M. Obaidat, B. A. Albiss, Y. Haik, Magnetic nanoparticles: Surface effects and properties related to biomedicine applications, International Journal of Molecular Sciences 14 (11) (2013) 21266. 